\title{
Technical Note Errata Erratorum: The Nature of the Quaternion
}

\author{
Malcolm D. Shuster ${ }^{1}$ \\ original errata below as it should have appeared. \\ on page 365 . \\ Erratum No. 1: Equations (13) through (15) should read

$$
\begin{gathered}
{ }^{\varepsilon} \hat{\mathbf{e}}_{i}^{\prime}=\sum_{j=1}^{3} A_{i j}^{\varepsilon / \varepsilon \varepsilon} \hat{\mathbf{e}}_{j} \quad i=1,2,3 \\
{ }^{\varepsilon^{\prime}} \hat{\mathbf{e}}_{i}=A^{\varepsilon^{\prime} / \varepsilon \varepsilon} \hat{\mathbf{e}}_{i} \quad i=1,2,3 \\
{ }^{\varepsilon^{\prime}} \hat{\mathbf{e}}_{i}^{\prime}={ }^{\varepsilon} \hat{\mathbf{e}}_{i} \quad i=1,2,3
\end{gathered}
$$

The errata published earlier [1], unfortunately, contained errors. We repeat the

The article in question was published in The Journal of the Astronautical Sciences, Vol. 56, No. 3, July-September 2008, pp. 359-373. It contains two errata

Erratum No. 2: In the first sentence of the paragraph that follows equation (15) replace "From equation (9) with $\boldsymbol{a}$ replaced by $\hat{\boldsymbol{e}}_{k}$ and $\boldsymbol{b}$ replaced by $\hat{\boldsymbol{e}}_{\boldsymbol{k}}$ " with "From equation (9) with $\boldsymbol{a}$ replaced by $\hat{\boldsymbol{e}}_{k}$ and $\boldsymbol{b}$ replaced by $\hat{\boldsymbol{e}}_{k}^{\prime}$."

The author would also like to acknowledge the generous help of Dr. John L. Crassidis of the University at Buffalo, State University of New York, for shepherding this work through final publication.

\section{References}

[1] SHUSTER, M.D. "Technical Note, Errata: The Nature of the Quaternion," The Journal of the Astronautical Sciences, Vol. 56, No. 4, Oct.-Dec. 2008, p. 621.

${ }^{1}$ Director of Research, Acme Spacecraft Company, 13017 Wisteria Drive, Box 328, Germantown, Maryland, 20874. Website: http://home.comcast.net/ mdshuster/. 